Article

\title{
Stability Criteria for Input Filter Design in Converters with CPL: Applications in Sliding Mode Controlled Power Systems
}

\author{
Jorge Luis Anderson Azzano* (), Jerónimo J. Moré $\mathbb{C}^{\mathbb{D}}$ and Paul F. Puleston \\ Instituto LEICI, Facultad de Ingeniería UNLP_CONICET, B1900 La Plata, Argentina; \\ jmore@ing.unlp.edu.ar (J.J.M.); puleston@ing.unlp.edu.ar (P.F.P.) \\ * Correspondence: anderson.jorgeluis@gmail.com
}

Received: 30 September 2019; Accepted: 21 October 2019; Published: 24 October 2019

check for updates

\begin{abstract}
Microgrids are versatile systems for integration of renewable energy sources and non-conventional storage devices. Sliding Mode techniques grant excellent features of robustness controlling power conditioning systems, making them highly suitable for microgrid applications. However, problems may arise when a converter is set to behave as a Constant Power Load (CPL). These issues manifest in the stability of internal dynamics (or Zero Dynamics), which is determined by the input filter of the power module. In this paper, a special Lyapunov analysis is conducted to address the nonlinear internal dynamics of SM controlled power modules with CPL. It takes advantage of a Liérnad-type description, establishing stability conditions and providing a secure operation region. These conditions are translated into conductance and invariant region diagrams, turning them into tools for the design of power module filters.
\end{abstract}

Keywords: Lyapunov-based filter design; constant power load; Sliding Mode controlled power module; zero dynamics stability

\section{Introduction}

Microgrids can be succinctly understood as an interactive hybrid system that combines distributed generation modules, storage modules and loads. The number and nature of these modules, as well as the microgrid topologies, vary greatly [1-3]. Presently, the modules interconnection is predominantly done using AC buses, but DC microgrids are expected to increase in the coming years [4]. In particular, DC microgrids are very versatile for integration of renewable energy sources and non conventional storage devices, such as photovoltaic panels, wind turbines, lithium batteries, flow batteries, supercapacitors, fuel cells and many others (see Figure 1). The increasing development of power electronics and novel control techniques have favoured their implementation, making it possible to obtain higher efficiency and reliability. Furthermore, distribution power systems have been widespread in several applications due to their flexibility features and reduced size and cost.

In this growing scenario, where a wide range of new topologies is being developed, technological challenges are continuously arising, many of them at the level of the modules power converters and their related controllers. The nonlinear behaviour of power converters in these systems often makes conventional linear control techniques not sufficiently effective. In addition, the existence of uncertainties and system disturbances require the development of robust controllers to ensure system stability and efficiency. In this context, Sliding Mode (SM) control have been increasingly accepted for controlling power conditioning systems [5-9]. These control techniques proved to be particularly suitable for electronic converters, granting excellent features of robustness and finite-time convergence, which make them highly appropriate for microgrid applications [10-14]. 


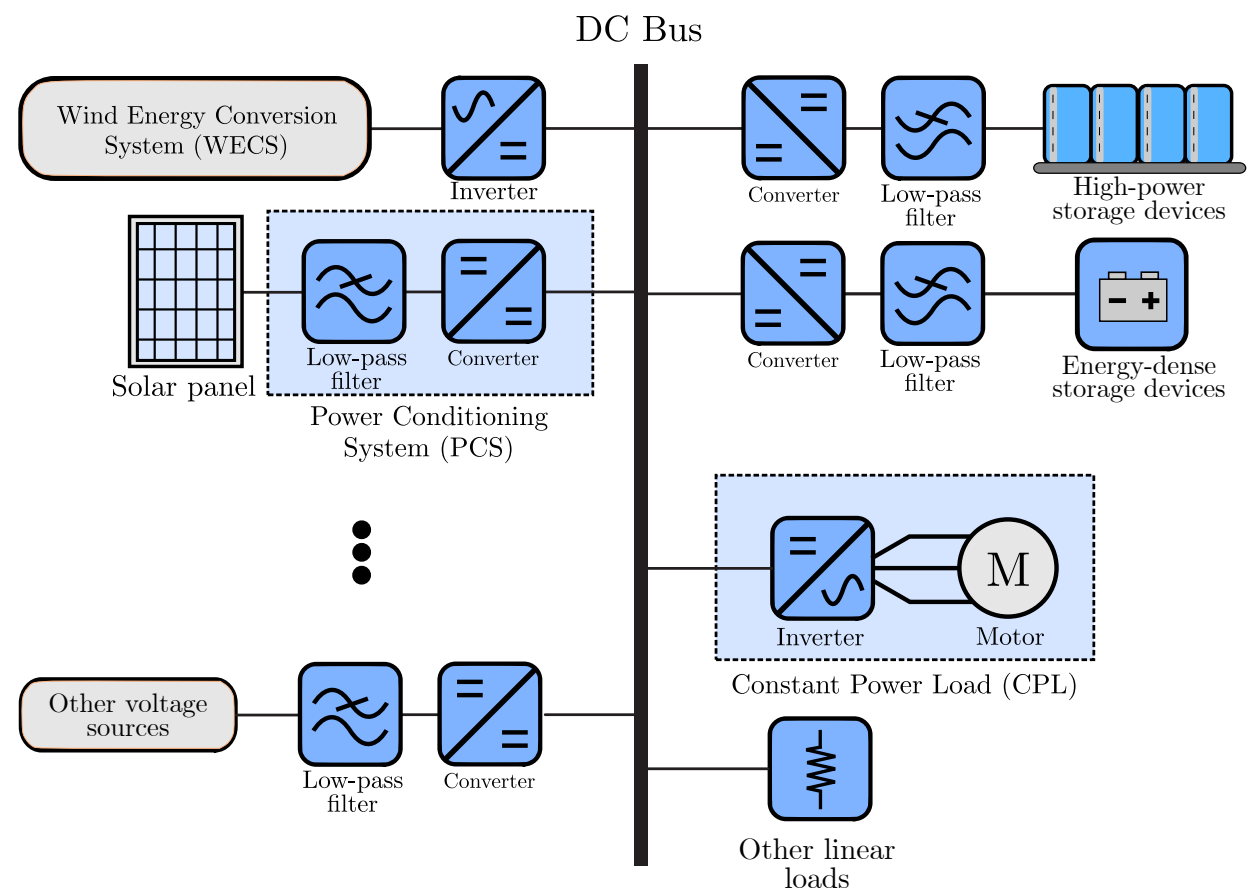

Figure 1. Illustrative representation of a DC microgrid combining renewable modules, non conventional storage modules and constant power load.

However, there are certain issues to be aware of at the moment of designing and implementing those power modules. Effectively, it has been extensively reported in the literature that, when a controlled power system tightly regulates energy supplied to a DC bus, the converter behaves as a Constant Power Load (CPL) for the upstream source [15-17]. Such CPL acts as a negative impedance, i.e., an increase in its terminal voltage results in a decrease in its current (and vice versa), which undermines the stability margins of the system.

In the case of SM controlled power systems, these issues are manifested in the stability of the Zero Dynamics (ZD). This internal dynamics is determined by the input filter of the power module, which exists to protect the source or the upstream device against switching harmonics propagation. If ZD is not carefully analysed and its stability is not thoroughly addressed during the design of the controlled power system, then performance and robustness could be severely compromised, causing undesired oscillations or even system failures.

Diverse criteria have been proposed for the analysis of input filters dynamics with CPLs in DC microgrids. The most widely found in the literature is the small-signal criterion by eigenvalue analysis through system linearization $[17,18]$. Also, several methods have been presented using Nyquist approaches, for instance, Gain margin and Phase margin criterion [19], Middlebrook's criterion [20] and the opposing argument criterion [21] among the most important ones. Moreover, in [22] a passivity-based criterion is proposed for a LC filter and in [23] stability conditions are found taking into account parameters variations through bifurcation analysis.

Those methods do not guarantee large-signal stability and give no precise information about the delimitation of a stable region for secure operation of the power module [21]. So as a contribution to this field, in this paper, a special Lyapunov analysis is conducted to address the nonlinear internal dynamics of SM controlled power modules with CPL. This study is performed taking advantage of a transformation to a Liérnad-type description, establishing sufficient conditions to define the stability of the nonlinear system and providing a secure operation region. These conditions are, subsequently, condensed into conductance and invariant region diagrams, which are proposed as versatile tools for the design of power module filters.

The paper is organised as follows. In Section 2, the proposed input filter design method for power converters with CPL is developed. It is divided into two main parts. The first one, Section 2.1, 
deals with the search of the stability conditions via Liénard-based nonlinear Lyapunov approach. Then, the second part, Section 2.2, translates those conditions into appropriate diagrams, based on which the filter design procedure will be grounded. In Section 3, simulation results are presented and analysed, considering as application case a SM controlled Boost convert with second-order filter. Finally, in Section 4, conclusions and future lines of research are discussed.

\section{Development of the Proposed Input Filter Design Method for Power Converters with CPL}

Two steps are required to develop the proposed method for input filter design. The first one is to obtain sufficient conditions for the zero dynamics (ZD) stability, over the full nonlinear operation range of SM controlled converters with CPL. Based on those conditions, the second step consists of elaborating a filter design criteria with the help of ad hoc conductance diagram description.

In Figure 2, a schematic diagram showing the topology of the power module under study is presented.

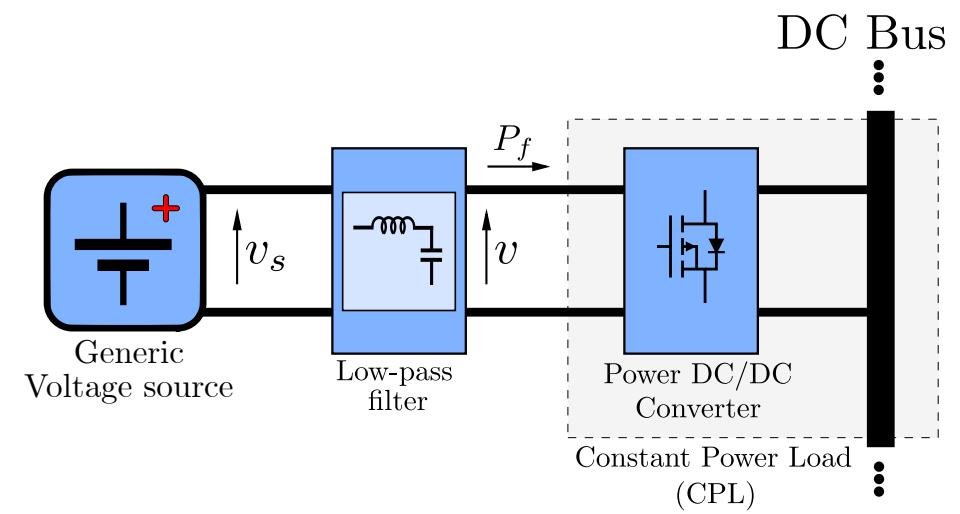

Figure 2. Schematic diagram of the Power Conditioning System (PCS) employed for the ZD analysis.

In the following subsections, both aforementioned steps are treated in detail.

\subsection{Search of Stability Conditions via Liénard Based Nonlinear Lyapunov Approach}

In this subsection, the ZD is thoroughly studied and sufficient conditions are obtained by mean of nonlinear Lyapunov analysis. For the sake of clarity, a path of progressive complexity is followed. Initially, a nonlinear Lyapunov approach is applied to a simple topology based on a first-order capacitive filter, and a sufficient condition for its stability is obtained. Then, the study is broadened to a widely-used second-order LC filter, describing the system in a Liénard form and analyzing its stability through a special energy-like Lyapunov function.

\subsubsection{First-Order Capacitive Filter Topology}

The electrical circuit of the capacitive filter supplying a constant power $P_{f}$ is shown in Figure 3. Its electrical model is defined by the first order differential equation:

$$
C_{f} \dot{v}=\frac{1}{R_{s}} V_{o c}-\frac{1}{R_{s}} v-\frac{P_{f}}{v}, \quad \forall v>0
$$

where $C_{f}$ is the filter capacitance, $V_{o c}$ is the open circuit source voltage, $R_{s}$ is the internal source resistance and $v$ the voltage across the capacitor. The nonlinear term $\frac{P_{f}}{v}$, corresponding to the CPL current, acts as a negative impedance, when the voltage $v$ is increased the filter output current is decreased. This negative impedance reduces the equivalent resistance of the system, leading to a reduction of the stability margin. 


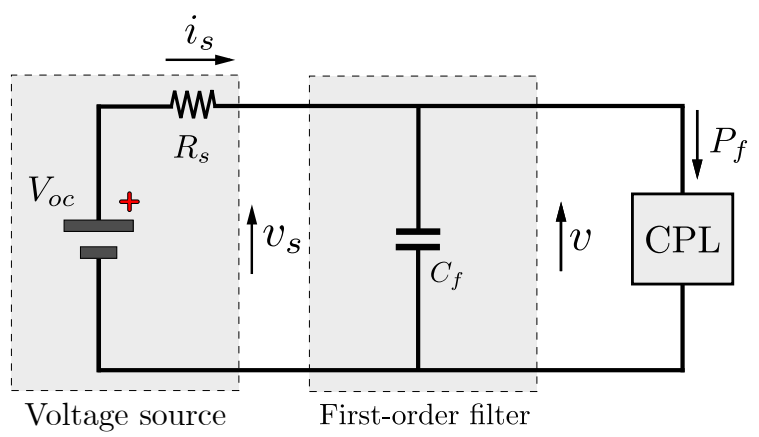

Figure 3. Scheme of the equivalent electrical circuit of the first-order input filter with CPL.

From (1), the well-known condition for the existence of equilibrium in CPL is [24]:

$$
P_{f} \leq \frac{V_{o c}^{2}}{4 R_{s}}=P_{f \max }
$$

which if strictly satisfied, results in two solutions. The first one,

$$
v_{0}=\frac{V_{o c}}{2}+\frac{1}{2} \sqrt{V_{o c}^{2}-4 P_{f} R_{s}}
$$

is the equilibrium capacitor voltage $v_{0}$, that defines the equilibrium point of operation of the power module. The second one,

$$
v_{l i m}=\frac{V_{o c}}{2}-\frac{1}{2} \sqrt{V_{o c}^{2}-4 P_{f} R_{s}}
$$

has no physical meaning, and represents a voltage limit for the system stability. Additionally, by multiplying (3) by (4), it is straightforward to obtain $v_{l i m}=\frac{P_{f} R_{s}}{v_{0}}$ (the operation equilibrium, given by $v_{0}$, and the stability limit, determined by $v_{l i m}$, can be visualised further in Figure 4 , at the end of this subsection).

As previously discussed, traditional stability study of the system described by (1) has been addressed by linearization, determining the local stability near the equilibrium voltage $v_{0}$ through eigenvalue analysis. In accordance with this conventional analysis, if $P_{f}$ satisfies:

$$
P_{f}<\frac{v_{0}^{2}}{R_{s}}
$$

then the eigenvalues of the linearised system around the equilibrium point have negative real part and so the trajectories of the system converge to the equilibrium voltage $v_{0}$, for close enough initial conditions.

It is well known that this linear analysis only ensures the stability for trajectories sufficiently close to $v_{0}$, but gives no information about the attraction region. In fact, it will be shown that a power system whose eigenvalues analysis results are stable, would in practice become unstable if voltage $v$ drops below the stability limit voltage $v_{\text {lim }}$.

Effectively, through the nonlinear Lyapunov analysis presented in the sequel, it is possible to completely define the system stability, allowing establishing the actual range of $v$ where secure operation is guaranteed.

To this end, in this simple first case study, the equilibrium point is translated to the origin through the state transformation: 


$$
z=v-v_{0}
$$

it leads to:

$$
\dot{z}=-a(z)
$$

with

$$
a(z)=\frac{1}{C_{f} R_{s}} z+\frac{P_{f}}{C_{f}}\left(\frac{1}{z+v_{0}}-\frac{1}{v_{0}}\right) .
$$

Now, the proposed energy-like Lyapunov function

$$
V(z)=\int_{0}^{z} a(\xi) d \xi
$$

is locally positive-definite if condition

$$
z a(z)>0
$$

is satisfied for some interval that includes the origin. Therefore, it follows that the first derivative of $V$ is equal to:

$$
\dot{V}(z)=-a(z)^{2}
$$

which is definite-negative for $a(z) \neq 0$.

The Lyapunov function, defined in (9), is shown in Figure 4 , with $z_{0}=0$ and $z_{\text {lim }}=v_{\text {lim }}-v_{0}$ obtained from (3) and (4), respectively, in combination with (6). As can be appreciated, the local maximum and minimum of the function $V(z)$ are at $z_{l i m}$ and $z_{0}$ (corresponding to capacitor voltages $v=v_{\text {lim }}$ and $v=v_{0}$, respectively). These values of $z$ are zeros of the function $\dot{V}(z)$, i.e., zeros of $a(z)$, and thus zeros of $\dot{z}$ (see (7)).

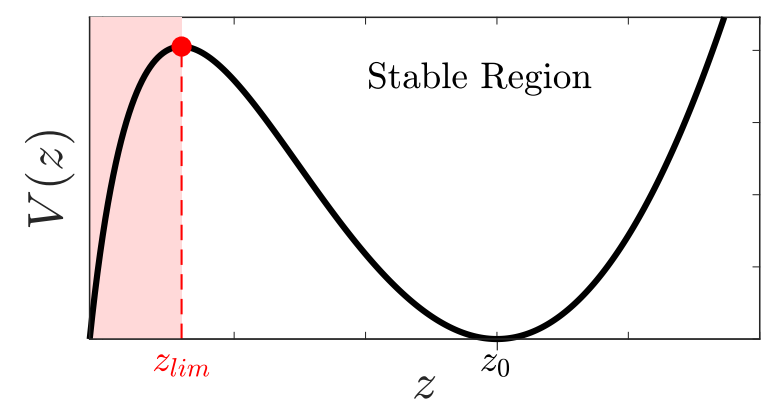

Figure 4. Energy-like Lyapunov function for first order filter study.

Stability condition in terms of conductances. It can straightforwardly obtained by computing inequality (10) as a function of the voltage $v$ :

$$
g_{0}\left(P_{f}, v\right)=\frac{P_{f}}{v_{0}} \frac{1}{v}<\frac{1}{R_{s}}=g_{s}
$$

where $g_{0}$ is an equivalent output conductance viewed from the filter and $g_{s}$ is the internal conductance of the voltage source. 
Stable operation region $\mathcal{S}$ for first-order filter. It should be remarked that, unlike condition (5) obtained via eigenvalue linear analysis, the condition given by (12) defines the stable region of the first-order filter, fully determining the admissible range for the capacitor voltage $v$. It establishes that if the voltage $v$ satisfies:

$$
v>v_{\text {lim }}=\frac{P_{f} R_{s}}{v_{0}} \text { with } P_{f} \leq P_{f \max }
$$

all system trajectories converge to the equilibrium voltage $v_{0}$. Otherwise, the system trajectories become unstable.

\subsubsection{Second-Order LC Filter Topology}

Founded on the previous approach, in this subsection, the Zero Dynamics stability of the power module is studied for widespread second-order input filters. To this end, nonlinear Lyapunov analysis is therefore employed to obtain stability conditions in terms of equivalent conductances. Then, these conditions will help to determine an invariant region where secure operation of power modules will be completely guaranteed.

Regretfully, this is not as direct as it is for the first-order capacitive filter. To be able to perform the analysis, in this work, the ZD is firstly rewritten into a special Liénard-type form [25], by means of a linear transformation. This allows a suitable energy-like Lyapunov function capable of dealing with this nonlinear topology.

Figure 5 shows a schematic model of the second-order LC filter connected to the converter acting as a constant power load.

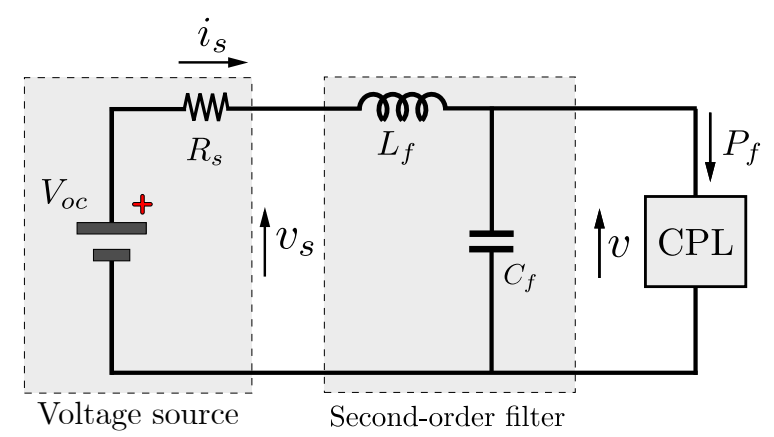

Figure 5. Scheme of the equivalent electrical circuit of the second-order input filter with CPL.

The system is described by:

$$
\left\{\begin{array}{l}
L_{f} \cdot i_{s}=-v-R_{s} \cdot i_{s}+V_{o c} \\
C_{f} \cdot v=i_{s}-\frac{P f}{v}
\end{array}\right.
$$

The above system presents an operation equilibrium point, defined by both the equilibrium source current $i_{s 0}$ and (if (2) holds) the equilibrium filter voltage $v_{0}$ :

$$
\begin{aligned}
& i_{s 0}=\frac{P_{f}}{v_{0}} \\
& v_{0}=\frac{V_{o c}}{2}+\frac{1}{2} \sqrt{V_{o c}^{2}-4 P_{f} R_{s}}
\end{aligned}
$$

where the latter is the solution with physical meaning for the power system operation, whereas there exists a second solution 


$$
v_{\text {lim }}=\frac{V_{o c}}{2}-\frac{1}{2} \sqrt{V_{o c}^{2}-4 P_{f} R_{s}}=\frac{P_{f} R_{s}}{v_{0}}
$$

which again represents a voltage limit for the system stability.

Analogously to the previous case, the equilibrium point is shifted to the origin by redefining the system states as:

$$
\begin{aligned}
& x_{1}=i_{s}-i_{s 0} \\
& x_{2}=v-v_{0},
\end{aligned}
$$

leading to the transformed system:

$$
\left\{\begin{array}{l}
\dot{x}_{1}=-\frac{R_{s}}{L_{f}} x_{1}-\frac{1}{L_{f}} x_{2} \\
\dot{x}_{2}=\frac{1}{C_{f}} x_{1}+\frac{P_{f}}{C_{f}}\left(\frac{1}{x_{2}+v_{0}}-\frac{1}{v_{0}}\right) .
\end{array}\right.
$$

To obtain a ZD representation convenient for the Lyapunov analysis, the following state linear transformation is proposed:

$$
\begin{aligned}
& z_{1}=\frac{1}{C_{f}} x_{1}+\frac{R_{s}}{L_{f}} x_{2} \\
& z_{2}=x_{2}
\end{aligned}
$$

and consequently, the transformed state system acquires the Liénard-type form [25]:

$$
\begin{aligned}
& \dot{z}_{1}=-a\left(z_{2}\right) \\
& \dot{z}_{2}=z_{1}-b\left(z_{2}\right)
\end{aligned}
$$

with

$$
\begin{aligned}
& a\left(z_{2}\right)=\frac{1}{L_{f} C_{f}} \cdot z_{2}+\frac{R_{s} P_{f}}{L_{f} C_{f}}\left(\frac{1}{z_{2}+v_{0}}-\frac{1}{v_{0}}\right) \\
& b\left(z_{2}\right)=\frac{R_{s}}{L_{f}} \cdot z_{2}+\frac{P_{f}}{C_{f}}\left(\frac{1}{z_{2}+v_{0}}-\frac{1}{v_{0}}\right) .
\end{aligned}
$$

Now, it can be proposed the appropriate energy-like Lyapunov function, depending on new states $z_{1}$ and $z_{2}$ :

$$
V\left(z_{1}, z_{2}\right)=\frac{1}{2} \cdot z_{1}^{2}+\int_{0}^{z_{2}} a(\xi) d \xi
$$

with $V(0,0)=0$. Then, similar to condition (10), if $z_{2}$ satisfies:

$$
z_{2} a\left(z_{2}\right)>0
$$

the integral term will be positive, and in consequence, $V\left(z_{1}, z_{2}\right)$ will be positive-definite for some region around the origin. 
Next, the first derivative of $V$ is straightly computed as:

$$
\begin{aligned}
\dot{V}\left(z_{1}, z_{2}\right)= & z_{1} \dot{z}_{1}+a\left(z_{2}\right) \dot{z}_{2}=-z_{1} a\left(z_{2}\right)+z_{1} a\left(z_{2}\right)-a\left(z_{2}\right) b\left(z_{2}\right) \\
\Longrightarrow \dot{V}\left(z_{1}, z_{2}\right) & =-a\left(z_{2}\right) b\left(z_{2}\right)
\end{aligned}
$$

which means that $\dot{V}$ will be locally negative-definite if it satisfies $a\left(z_{2}\right) b\left(z_{2}\right)>0$ for some interval around the origin. Therefore, taking into account condition (28), it leads to:

$$
\begin{aligned}
a\left(z_{2}\right) b\left(z_{2}\right)>0 & \Longrightarrow a\left(z_{2}\right)^{2} \cdot z_{2} \cdot b\left(z_{2}\right)>0 \\
& \Longleftrightarrow z_{2} \cdot b\left(z_{2}\right)>0 .
\end{aligned}
$$

Hereunder, the Lyapunov conditions (28) and (31) will be rewritten in terms of equivalent conductances, preparing the ground for the completion of the proposed design method in the following subsection.

First Lyapunov condition in terms of conductances. It is obtained combining condition (28) with (25), giving:

$$
\begin{gathered}
\frac{1}{L_{f} C_{f}} z_{2}^{2}\left(\frac{1}{R_{s}}-\frac{P_{f}}{\left(z_{2}+v_{0}\right) v_{0}}\right)>0 \\
\Longrightarrow g_{0}\left(P_{f}, v\right)=\frac{P_{f}}{v v_{0}}<\frac{1}{R_{s}}=g_{s}
\end{gathered}
$$

where similarly to (12), $g_{0}$ is the equivalent output conductance viewed from the filter and $g_{s}$ is the internal conductance of the source.

Second Lyapunov condition in terms of conductances. It is computed from condition (26) and (31), resulting in:

$$
\begin{aligned}
& z_{2}^{2}\left(\frac{R_{s}}{L_{f}}-\frac{1}{C_{f}} \frac{P_{f}}{\left(z_{2}+v_{0}\right) v_{0}}\right)>0 \\
\Longrightarrow & g_{0}\left(P_{f}, v\right)=\frac{P_{f}}{v v_{0}}<\frac{R_{s} C_{f}}{L_{f}}=g_{l c}
\end{aligned}
$$

where the new equivalent conductance $g_{l c}$ is called filter conductance.

Furthermore, both conditions can be summarised in one inequality as:

$$
g_{0}\left(P_{f}, v\right)<\min \left(g_{s} ; g_{l c}\right)
$$

or, similarly to the first-order filter, in terms of voltage $v$, as:

$$
v>v_{\text {min }}=\max \left(v_{\text {lim }}=\frac{P_{f} R_{s}}{v_{0}} ; \frac{P_{f} L_{f}}{R_{s} C_{f} v_{0}}\right) \text { with } P_{f} \leq P_{f \max }
$$

However, unlike the first-order case, the above condition (alternatively (36) or (33)-(35)) is not sufficient to ensure the ZD stable behaviour, because not all trajectories will necessarily converge to $\left(i_{s 0}, v_{0}\right)$ for any initial conditions $v>v_{\text {min }}$. In fact, with a second-order filter, the stable region also depends on the inductor current $i_{s}$.

Nevertheless, (37) does guarantee the existence of an invariant region around the equilibrium point, within which all system trajectories would converge to the desired equilibrium. Moreover, in contrast to the eigenvalues approach, following the subsequent analysis this stable region can be fully determined and eventually used as a helping tool for the filter design. 
Stable operation region $\mathcal{S}$ for second-order filter. Condition (37) is therefore used to define such invariant region in the ZD state plane, where performance and secure operation of the SM controlled power system can be ensured.

For better interpretation, Figure 6 schematically depicts the Lyapunov function $V\left(z_{1}, z_{2}\right)$, including both equilibrium solutions, these are the the desired equilibrium point of operation, $p_{0}=\left(i_{s 0}, v_{0}\right)$ in green, and the limit point $p_{\text {lim }}=\left(i_{s 0}, v_{\text {lim }}\right)$ in red. The rationale behind the proposed procedure is quite simple. A basin of attraction targeting to $p_{0}$ must be devised by intersecting $V\left(z_{1}, z_{2}\right)$ with an appropriate horizontal plane, Plane $_{\text {invar }}$.

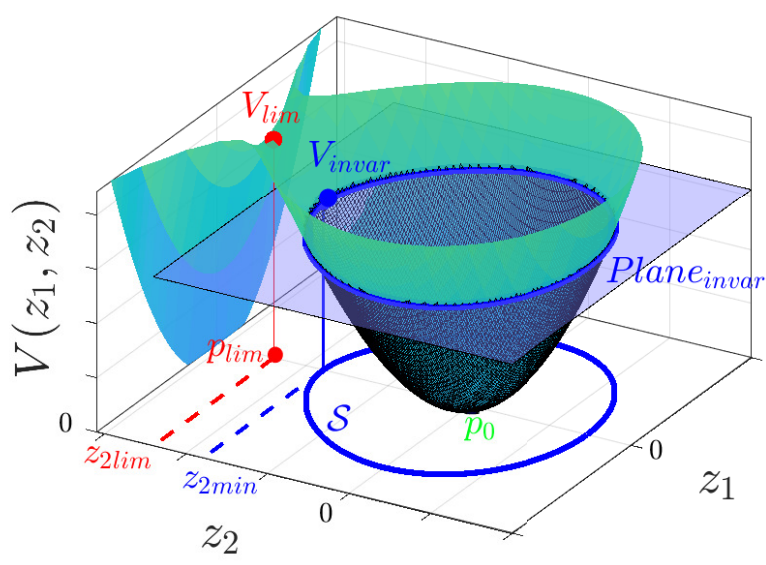

(a) Lyapunov function $V\left(z_{1}, z_{2}\right)$.

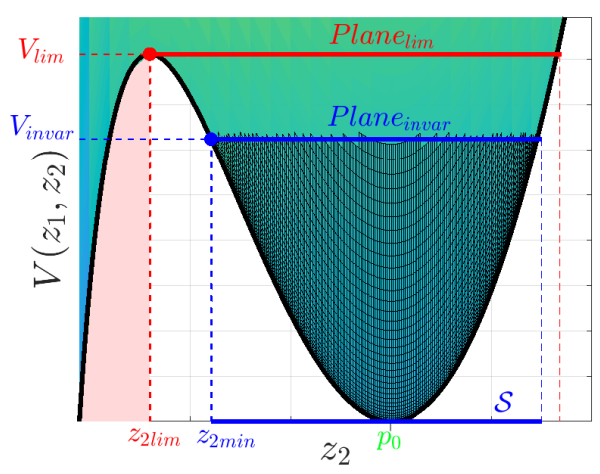

(b) Lyapunov function $V\left(z_{1}, z_{2}\right)$. Lateral view.

Figure 6. Illustration of the invariant region obtained through Lyapunov stability conditions.

It can be easily proved that the limit point $p_{\text {lim }}$ is an unstable equilibrium, hence it defines a plane, Plane $e_{\text {lim }}$ (given by $V\left(z_{1}, z_{2}\right)=V_{\text {lim }}$, with constant $V_{\text {lim }}=V\left(p_{\text {lim }}\right)$ ), which naturally is an upper bound for Plane invar $_{\text {. Moreover, Plane }}$ lim delimited a basin-shaped, yet not necessarily attractive, Lyapunov function.

Therefore, to ensure attractiveness to the desired equilibrium, the lower (or equal) Plane invar $_{\text {r }}$ should be obtained such that, below it, Lyapunov conditions hold (or, equivalently, condition (37) holds). Then, the proposed invariant region (in blue solid line) is delimited by the intersection of

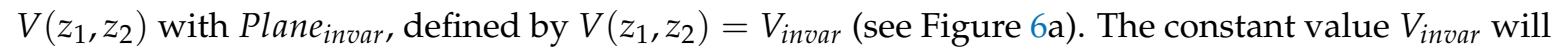
be computed below using $v_{\text {min }}$, such that $\dot{V}$ results negative for all $\left(z_{1}, z_{2}\right): V\left(z_{1}, z_{2}\right)<V_{\text {invar }}$, thus the basin of attraction is attained and all ZD trajectories which start below Plane $e_{\text {invar }}$ converge to $p_{0}$. Therefore, the stable invariant region can be formally defined

$$
\forall\left(z_{1}, z_{2}\right) \in \mathcal{S}: V\left(z_{1}, z_{2}\right)<V_{\text {invar }}
$$

where the constant $V_{\text {invar }}$ is computed according to (27):

$$
\begin{gathered}
\left.V_{\text {invar }}=\min \left(V\left(z_{1}, z_{2, \min }\right)\right)=\min \left(\frac{1}{2} z_{1}^{2}+\int_{0}^{z_{2, \text { min }}} a(\xi) d \xi\right)\right) \\
\Longrightarrow V_{\text {invar }}=\int_{0}^{z_{2, \min }} a(\xi) d \xi
\end{gathered}
$$

with

$$
z_{2, \min }=v_{\min }-v_{0}
$$


Please note that the way $v_{\min }=\max \left(v_{l i m} ; \frac{P_{f} L_{f}}{R_{s} C_{f} v_{0}}\right)$ is defined in (37), effectively implies that Plane $_{\text {lim }}$ is the upper limit plane for the Plane $i n v a r$.

\subsection{Proposed Filter Design Criteria through Conductance Diagram Description}

The second step to conclude the method for input filter design is developed in this subsection. It is based on translating the previously obtained stability conditions into appropriate conductance diagrams. These diagrams, complemented with those of the stable operation region of the power module, are then proposed as practical tools for systematic design.

\subsubsection{Design Criterion for the First-Order Input Filter}

The case of the power module with a first-order input filter is relatively simple and can be straightforwardly designed with the assistance of condition (12) (or, equivalently, (13)). Therefore, the main contribution of this case to the paper is not the design itself, but the introduction and interpretation of the conductance diagrams, familiarising the reader with their use in the framework of a filter design procedure, aiming to the more interesting second-order topology to come.

For the case under study, the condition arrived in (12) provides a relation between the output conductance $g_{0}$ and the source conductance $g_{s}$ for the stability of the ZD system described in (1). This relation can be compared for different values of the output power $P_{f}$ in a conductance diagram, where a stable operation region can be established.

Figure 7 presents the conductance diagram for a voltage source with open-circuit voltage $V_{o c}=24 \mathrm{~V}$ and internal resistance $R_{S}=0.144 \Omega$. The diagram is constructed using the conditions (12)-(13), obtained from the procedure described in Section 2.1.1. Each hyperbola (dashed black lines) represents an output conductance $g_{0}$ for a specific value of power $P_{f}$. For the sake of illustration, the conductance $g_{0}$ for a given power $P_{f}=P_{\text {fnom }}$ is highlighted in thick black line. The upper limit curve corresponds to the maximum allowed power $P_{f \max }$, computed through (2).

In addition, the conductance $g_{0}$ at each equilibrium point (green line) is obtained as:

$$
g_{0}\left(P_{f}, v_{0}\right)=\frac{P_{f}}{v_{0}^{2}}
$$

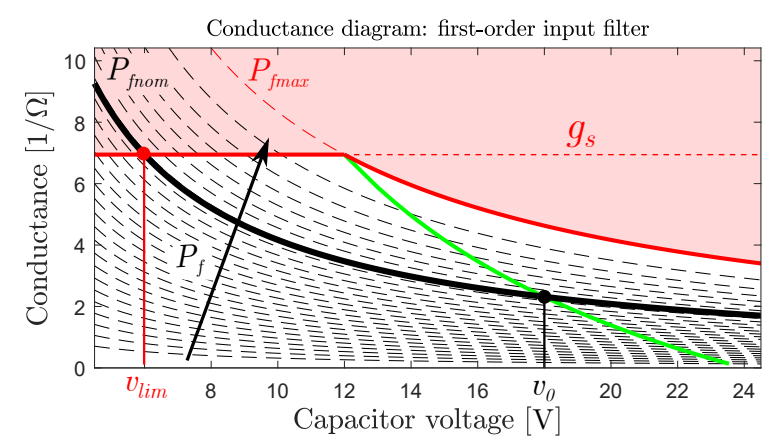

Figure 7. First-order filter conductance diagram for variable filter power $P_{f}$.

The intersection between the source conductance $g_{s}$ (horizontal red line) and each $g_{0}$ defines the limit voltage $v_{\text {lim }}$, which establishes the secure operation of the power system (the locus constituted by these points for different $P_{f}$ corresponds to the solid red segment). Then, the region of stable operation is delimited by the boundary conformed by the latter and the maximum allowed power, depicted in solid red line. All trajectories that start inside this region (white background in the figure) will converge to the equilibrium voltage $v_{0}$ (green line). Otherwise, they will become unstable. 
Please note that for the first order filter case the conductance diagram is defined by the source parameters $R_{S}$ and $V_{S}$, thus the stability region will not be altered by the selection of the filter capacitance $C_{f}$.

Therefore, from the above, the design procedure for this case study is direct. Firstly, knowing the desired cut-off frequency $f_{c}$ for the filter and $R_{s}$, capacitance $C_{f}$ is computed.

Then, from the stability region of the conductance diagram, for a desired power $P_{f}=P_{\text {fnom }}$ specified in the design, the admissible range of $v$ can be obtained. Alternatively, for a known admissible range of variation of the capacitor voltage $v$, an upper limit for the $P_{\text {fnom }}$ of the power module can be set. It can be appreciated in the conductance diagram that exists a trade-off between the selection of the operation power $P_{\text {fnom }}$ and the admissible variation range of $v$.

\subsubsection{Design Criterion for Second-Order Input Filter}

Building on the previous approach, in this subsection the design criterion for more prevalent second-order filter in converters with CPL is presented. As it was mentioned in Section 2.1.2, unlike the first-order case, the condition $v>v_{\min }$ given by (37) is necessary but not sufficient for the design. Therefore, the conductance diagram remains as a helpful design tool to determine and easily visualise the viable stable operation points. However, to ensure a design that provides wholly secure operation of the power module, it must be complemented with an invariant operation region diagram. This invariant region is constructed through the proposed procedure presented in Section 2.1.2 by means of (38)-(41). The value of the constant $V_{\text {invar, }}$, which defines the stable region, is computed from (39), using diverse values of filter power $P_{f}$ and filter parameters.

To facilitate the understanding of those diagrams for this topology, a descriptive analysis is presented below, together with illustrative diagrams of conductance and of the invariant region for different values of $P_{f}$, assuming fixed cut-off frequency $f_{c}$ and filter capacitor $C_{f}$.

As with the first-order case, the conductance diagram shown in Figure 8a is constructed through the set of Equations (32)-(37). However, now, the extra condition defined by (35), which strongly depends on the filter parameters, also needs to be considered.

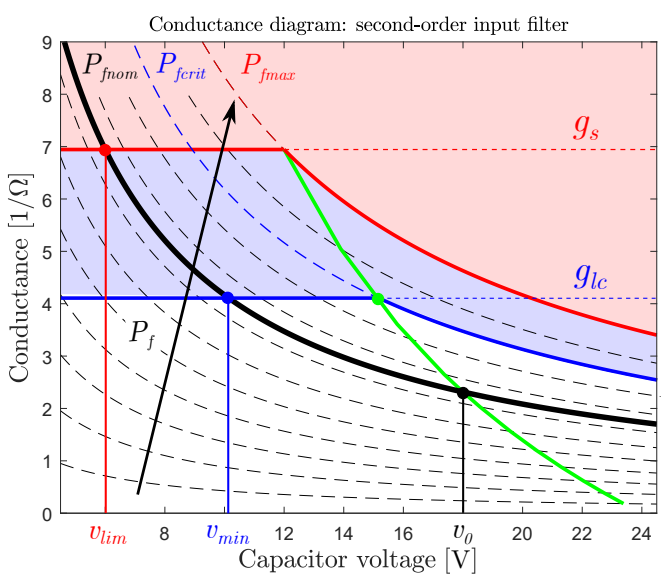

(a)

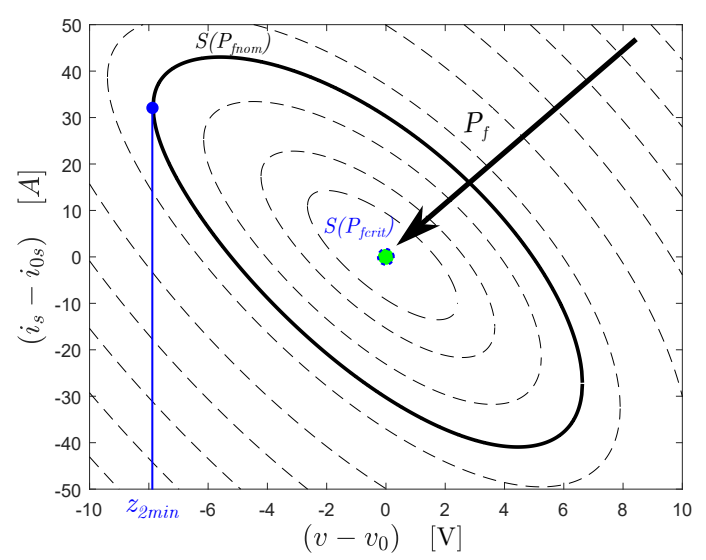

(b)

Figure 8. llustrative diagrams (a) of conductance and (b) of invariant region for second-order filter with variable power $P_{f}$ and fixed $f_{c}$ and $C_{f}$.

As in the previous analysis, the green line depicts the equilibrium locus, constituted by the conductance $g_{0}$ at each equilibrium point $g_{0}\left(P_{f}, v_{0}\right)=\frac{P_{f}}{v_{0}^{2}}$, for different $P_{f}$. However, in this case, the red solid line defined by the source conductance $g_{s}$ and $P_{f \max }$ is not a stability boundary. In fact, the conductance condition in this topology, set by inequality (36), depends not only on $g_{s}$, but also on the equivalent conductance $g_{l c}$ (dashed blue line). So, a more restrictive boundary (in thick solid blue 
line) arises, conformed by $\min \left(g_{s} ; g_{l c}\right)$ and a critical power $P_{f c r i t}$. The latter is an upper bound for $P_{f}$, beyond which the equilibrium points of the green locus are unstable. It can be computed by

$$
P_{f c r i t}=\frac{v_{0}^{2} R_{s} C_{f}}{L_{f}}
$$

Please note that the white-background region delimited by the blue boundary, does define the stable equilibria, hence it allows the visualisation of the admissible values of $P_{f}$ that can be selected as operation filter power.

Nevertheless, it does not provide per se a region that secures stable operation, as it was for the first-order filter. Thus, to take into account in the design procedure the admissible variation ranges of $v$ and $i_{s}$, the assistance of the invariant region diagram is required. In Figure $8 \mathbf{b}$ invariant regions $\mathcal{S}$ are plotted, parametrised for different values of filter power $P_{f}$, with their boundaries computed as $V\left(z_{1}, z_{2}\right)=V_{\text {invar }}$ in accordance with (38)-(41).

The admissible ranges of $v$ and $i_{s}$ strongly depend on the selected operation power $P_{f}$. Note the existing trade-off for the design. The higher the selected $P_{f}$, getting closer to the $P_{f c r i t}$, the lower the invariant region, reducing the stability tolerance to admissible variations of $v$ and $i_{s}$ with respect to the nominal operation point.

In this framework, a sequential design procedure will be succinctly presented below, as an illustrative example of application.

- Firstly, the desired cut-off frequency $f_{c}$ is chosen to protect the source against switching harmonics propagation. It should be carefully selected, considering that a small value of $f_{c}$ could lead to large components sizing, which would imply a higher cost and weight. On the other hand, there is an upper bound for $f_{c}$ given by the switching converter frequency, whose practical higher limit is typically selected one decade below such switching frequency. In this case study, a $f_{c}$ value equals to $1 \mathrm{kHz}$ is taken.

- Next, the operation power $P_{f}$ is selected, in accordance with the power module requirements. In this example, the second-order input filter is designed for supplying an electrical power up to $P_{f}=750 \mathrm{~W}$ (solid black line in Figure 9a).

- The goal of the following step is to obtain the range of selectable values for capacitor $C_{f}$. These values are those which guarantee stable operation points for the $f_{c}$ and $P_{f}$ previously chosen. This step is easily fulfilled using the conductance diagram in Figure 9a, where $g_{l c}$ is plotted for different values of capacitor $C_{f}$ (blue horizontal lines).

It can be observed in Figure 9a that there exists a minimum value of capacitor $C_{f}$, equals to $0.65 \mathrm{mF}$, for which the equilibrium point of $P_{f}=750 \mathrm{~W}$ (black dot) would become unstable. So, designing $C_{f}$ greater than this so-called $C_{f \min }$ ensures that the stable equilibria region (white background zone) will contain the desired equilibrium point.

Therefore, from the conductance diagram, the range of selectable values for the filter capacitor can be set as $C_{f}>0.65 \mathrm{mF}=C_{f \min }$. If required, this value can be analytically computed through (35), as a function of the cut-off frequency:

$$
C_{f \min }=\frac{1}{2 \pi f_{c}} \frac{1}{v_{0}} \sqrt{\frac{P_{f}}{R_{s}}} .
$$

- Then, from those admissible values of $C_{f}$, one should be chosen, according to the required variation ranges of $v$ and $i_{s}$, which are contemplated in the specifications of the power module. This design step is conducted with the help of the invariant region diagram in Figure $9 b$, parametrised in terms of $C_{f}$. 


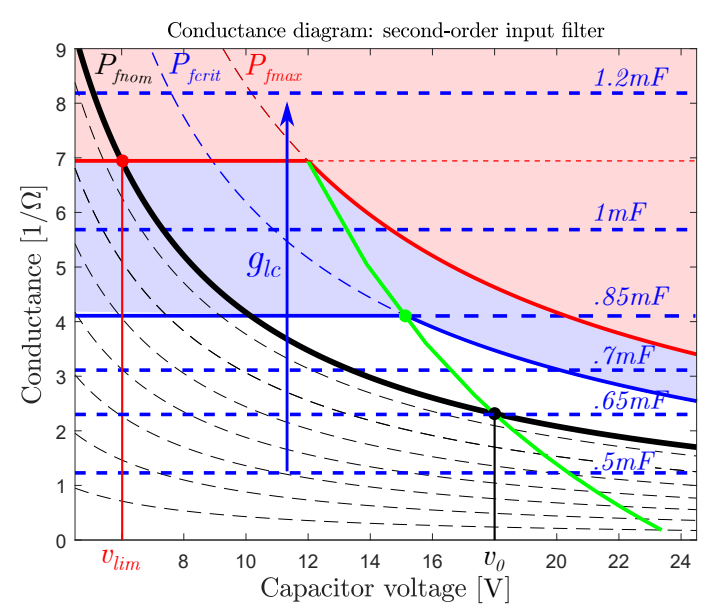

(a)

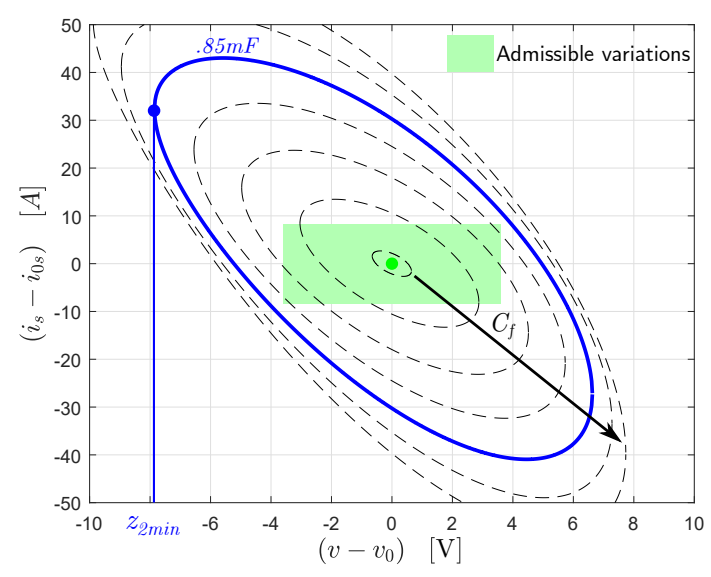

(b)

Figure 9. Design example: diagrams (a) of conductance and (b) of invariant region for second-order filter with variable filter capacitor $C_{f}$ and fixed $f_{c}=1 \mathrm{kHz}$ and $P_{f}=750 \mathrm{~W}$.

To illustrate the selection procedure, variations up to $\pm 20 \%$ for both voltage $v$ and current $i_{s}$, are considered admissible for the normal operation of the power module (represented by the green area Figure 9b).

Now, the proposed design criterion is simple. The smallest $C_{f}$ should be selected, such that its corresponding stable operation invariant region includes the admissible variation green area. Therefore, for this case study, $C_{f}=850 \mu \mathrm{F}$ is chosen (see curve in blue line).

Please note that there is a trade-off between the capacitor sizing and the allowed voltage $v$ and current $i_{s}$ variations. In this particular case, all capacitances larger than $850 \mu \mathrm{F}$ would provide secure stable operation to the power module.

- Finally, once the required $C_{f}$ was selected and knowing the desired $f_{c}$, the inductance $L_{f}$ can be easily obtained from:

$$
L_{f}=\left(\frac{1}{2 \pi f_{c}}\right)^{2} \cdot \frac{1}{C_{f}}
$$

which for this illustrative example, results $L_{f}=30 \mu \mathrm{H}$.

To conclude, it should be remarked that the previous procedure is just one of diverse possible ways to perform the filter design, which may differ depending on each particular case, available data and specifications.

\section{Application Case: SM Controlled Boost Converter with Second-Order Filter}

In this section, following the above proposed criteria, the ZD stability of a second-order input filter for a SM controlled boost converter-based power module is evaluated. An illustrative electrical diagram of the considered system is presented in Figure 10. The power module is intended to operate as a part of a microgrid, as the one presented in Figure 1, connected to a common DC Bus, in this case of $48 \mathrm{~V}$. In addition, the power module is fed by the power source $v_{s}\left(i_{s}\right)$, characterized by its open circuit voltage and internal resistance, which may represent different kinds of sources (i.e., Li-Ion batteries, Flow batteries, Fuel Cells, Supercapacitors, etc.). 


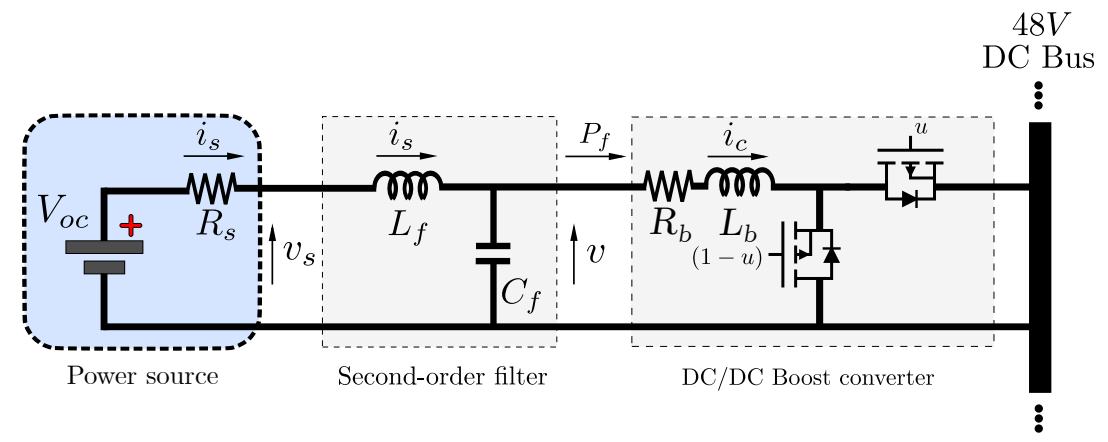

Figure 10. Electrical diagram of the PCS based on boost converter with second-order input filter.

In this particular case study, the power converter is assumed to be controlled by means of a Second Order Sliding Mode (SOSM) algorithm, designed to regulate a constant power to the DC bus (see details in Appendix A).

Under this CPL assumption, the filter is design following the criterion explained in Section 2.2.2. Then, in the sequel, to validate the proposed design method, the power system is evaluated under different initial conditions and filter power $P_{f}$.

Recapitulating, a cut-off frequency $f_{c}=1 \mathrm{kHz}$ and an operation filter power $P_{f}=750 \mathrm{~W}$ were selected. Next, the proposed design provided a filter capacitor of $C_{f}=850 \mu \mathrm{F}$, considering admissible voltage and current variations up to $\pm 20 \%$. Lastly, $L_{f}$ was computed using (45), giving $L_{f}=30 \mu \mathrm{H}$.

The complete set of system parameters employed for the subsequent simulations results are displayed in Table 1. It is important to remark that with this set of parameters, the resulting critical power $P_{\text {fcrit }}$ is of about $930 \mathrm{~W}$. If the system exceeds this power value, the equilibrium points become unstable (see Figure 9a).

Table 1. Parameters of the simulated power module.

\begin{tabular}{cccccc}
\hline \multicolumn{3}{c}{ System Parameters } & \multicolumn{2}{c}{ STA Parameters } \\
\hline$V_{o c}$ & $24 \mathrm{~V}$ & $f_{c}$ & $1 \mathrm{kHz}$ & $\beta$ & 200 \\
$R_{s}$ & $0.144 \Omega$ & $C_{f}$ & $0.85 \mathrm{mF}$ & $\alpha$ & $5 \times 10^{-3}$ \\
$P_{f}$ & $750 \mathrm{~W}$ & $L_{f}$ & $30 \mu \mathrm{H}$ & & \\
$L_{b}$ & $50 \mu \mathrm{H}$ & $R_{b}$ & $1 \mathrm{~m} \Omega$ & & \\
$f_{s}$ & $50 \mathrm{kHz}$ & $V_{d c}$ & $48 \mathrm{~V}$ & & \\
\hline
\end{tabular}

Then, to evaluate the system behaviour, several in-silico test were conducted. In the first place, the system is forced to operate under different values of constant power, until the critical power $P_{f c r i t}$ is reached. This results are shown in Figure 11.

Figure 11a presents the filter power evolution for such stepped power reference. It can be seen that the delivered power results equal to the reference, until the critical power $P_{\text {fcrit }}$ is surpassed ( $\sim 0.75 \mathrm{~s})$. At this moment, the $\mathrm{ZD}$ becomes unstable and the filter power collapses to zero.

Figure $11 \mathrm{~b}$ depicts the corresponding filter voltage and current. Please note that as the the filter power increases, the $v$ and $i_{s}$ variations during transients become bigger. Once the critical power is surpassed, the voltage drops to zero and the current saturates at its maximum $\left(i_{s}=V_{o c} / R_{S}\right)$.

The following set of tests aims to show that region $\mathcal{S}$, obtained via the proposed design, is an invariant region and that the inside trajectories converge to the desired equilibrium point. To this end, Figure 12 displays the trajectories in the $i_{s}-v$ plane for several initial conditions (red dots) placed near the boundary of $\mathcal{S}(750 \mathrm{~W})$ (blue curve). Effectively, as was previously discussed, it can be appreciated that this region ensures ZD stability of the SM controlled module, guaranteeing its secure operation. 


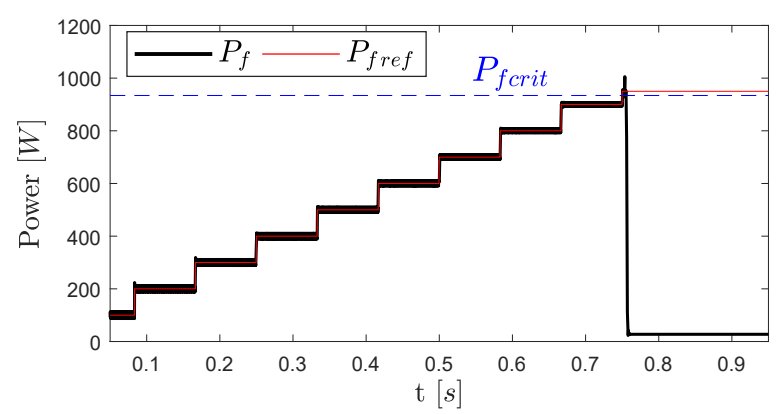

(a) Filter power evolution.
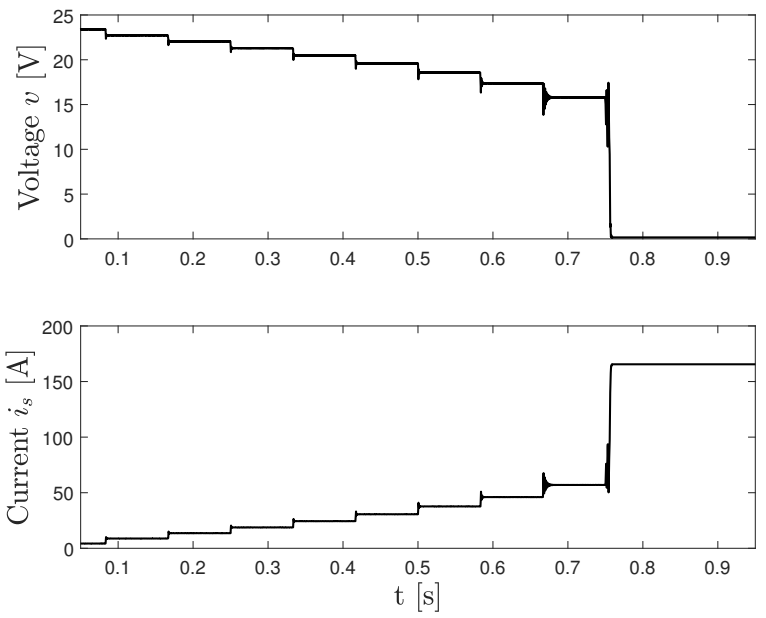

(b) Zero Dynamics states

Figure 11. Time response of the controlled power module for multiple reference steps.

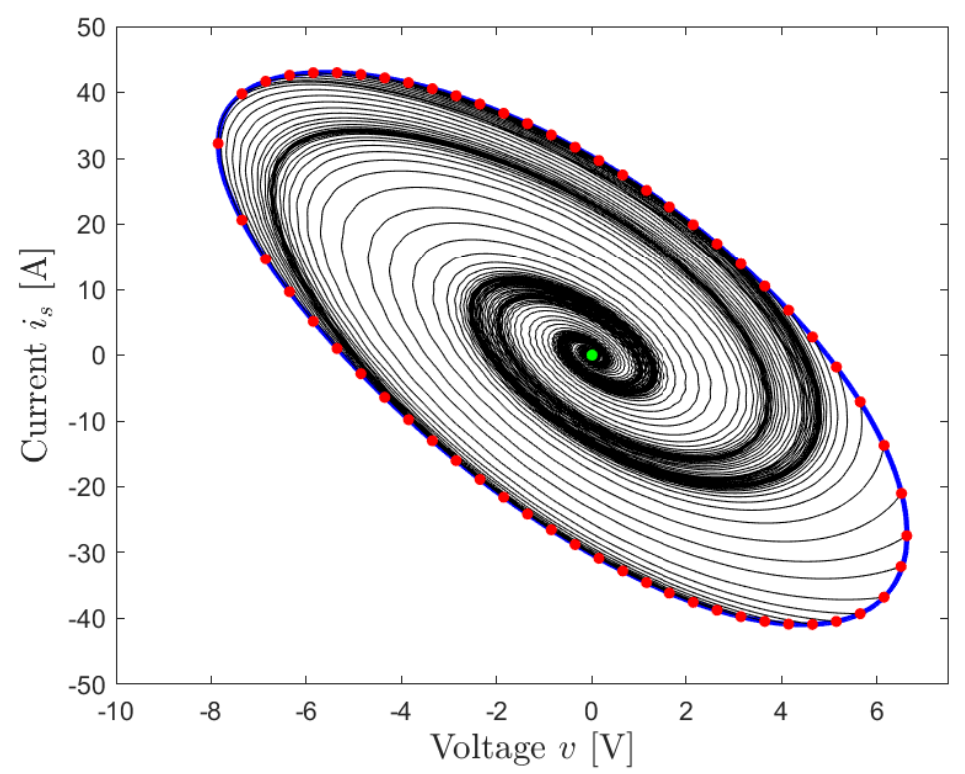

Figure 12. $i_{s}-v$ plane for stable trajectories with initial conditions inside invariant region $\mathcal{S}(750 \mathrm{~W})$.

The final test presented in Figure 13 is intended to illustrate the unstable behaviour for an initial condition outside the invariant region $\mathcal{S}(750 \mathrm{~W})$. The latter is plotted in blue line in the $i_{s}-v$ plane (top left figure in Figure 13). It can be observed in this example, as the ZD stability is not guaranteed, the system trajectory is not converging to equilibrium. It is important to stress that not all possible initial conditions outside this invariant region $\mathcal{S}$ result in unstable trajectories.

It is worth noticing that the SOSM-STA manages to reach the power reference of $750 \mathrm{~W}$ only for about $0.5 \mathrm{~ms}$ (see top right figure in Figure 13. Power reference in red line). As the ZD under this conditions results unstable, the controller is not able to remain over the sliding surface for a long time. Hence, the filter voltage $v$ drops to zero while the filter current saturates at its maximum $\left(i_{s}=V_{o c} / R_{s}\right)$, leading to a system failure. 

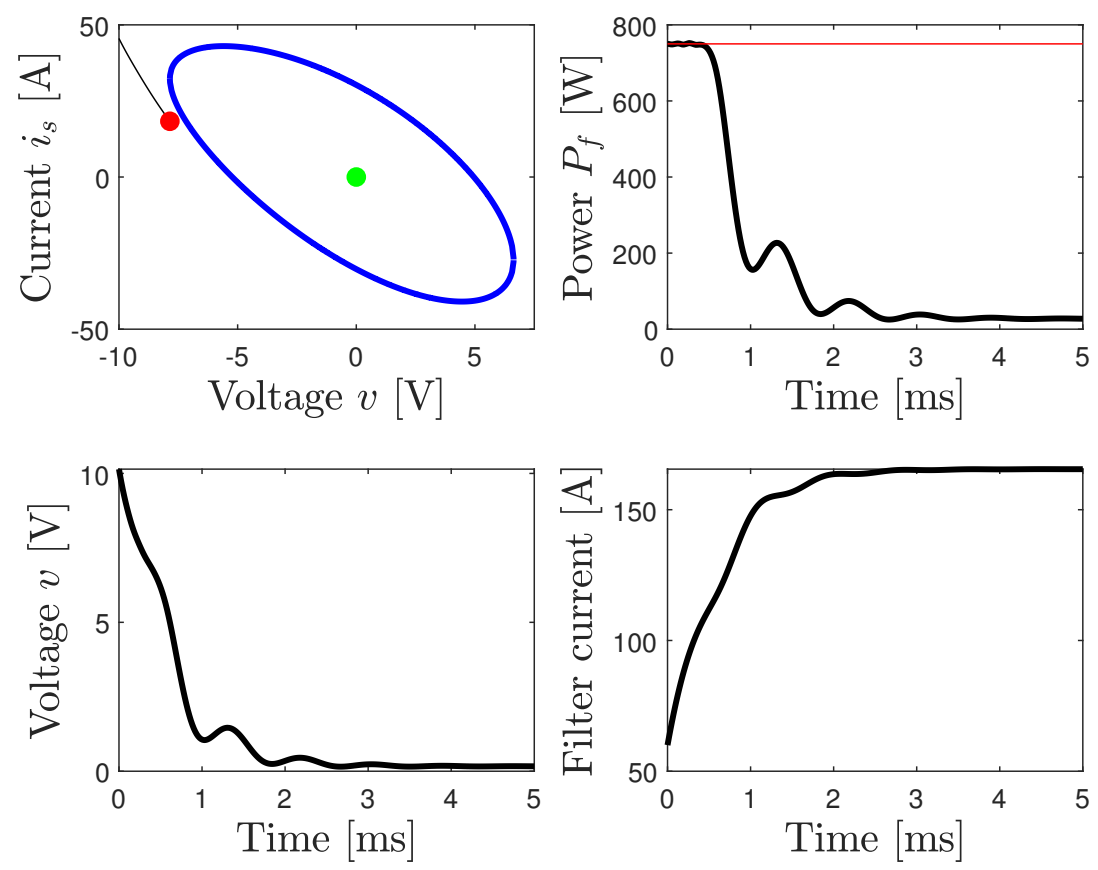

Figure 13. Unstable behaviour for initial condition outside invariant region $\mathcal{S}(750 \mathrm{~W})$.

\section{Conclusions}

This paper proposed design criteria for input filters operating in DC microgrid applications with CPL. The presented methods were aimed to ensure the stable operation of power modules controlled by Sliding Mode Control techniques. To this end, conductance and invariant region diagrams were proposed as practical tools for filter design. The use of these diagrams represents a handy visual assistance for the selection of the filter elements.

To develop this procedure, firstly, the Zero Dynamics of the SM controlled module were brought into a particular Liérnad-type form, through a special transformation. Then, stability conditions, in term of equivalent conductances, were obtained using a suitable energy-like Lyapunov function capable to deal with the ZD nonlinear structure. Finally, the proposed conductance diagram and invariant region were presented, allowing establishing a sequential filter design procedure.

With the purpose of validating the proposed methodology, a SM controlled Boost converter with a second-order filter was assessed by simulation. The output power of the module was assumed regulated by means of a Second Order Sliding Mode Super-Twisting Control. The module tests consisted on leading the system to different power condition until reaching critical power condition, where the system becomes unstable. Also, it was proved that, for initial conditions outside the proposed stable region, the operation of the SM controlled system was not guaranteed.

Future studies will be focused on extending the proposed methodology to nonlinear input power source. In addition, in the next stage of this research, to evaluate the design criteria against real unmodelled dynamics and perturbation, the implementation of experimental tests will be undertaken.

Author Contributions: Conceptualization, P.F.P.; formal analysis, J.L.A.A. and J.J.M.; investigation, J.L.A.A., J.J.M. and P.F.P.; methodology, J.L.A.A. and P.F.P.; project administration, P.F.P.; supervision, P.F.P.; validation, J.L.A.A. and J.J.M.

Funding: This research was funded by ANPCyT PICT N2015-2257 “Control, Electrónica e Instrumentación: Aplicaciones en Energías Alternativas y Bioingeniería”, LEICI, Fac. Ingeniería, UNLP. 2016-2019; CONICET PIP 112-2015-0100496CO "Electrónica de potencia y sistemas de control avanzado aplicados a fuentes de energía no convencionales", LEICI, Fac. Ingeniería, UNLP. 2016-2019 and UNLP Proyect 11/I217 "Electrónica de potencia y sistemas de control avanzado aplicados a fuentes de energía no convencionales", LEICI, Fac. Ingeniería, UNLP. 2016-2019.

Acknowledgments: This research was supported by the Universidad Nacional de La Plata (UNLP); the Fac. Ingeniería, UNLP; the Consejo Nacional de Investigaciones Científicas y Técnicas (CONICET) and the Agencia Nacional de Promoción Científica y Tecnológica (ANPCyT) from Argentina. 
Conflicts of Interest: The authors declare no conflict of interest. The funders had no role in the design of the study; in the collection, analyses, or interpretation of data; in the writing of the manuscript, or in the decision to publish the results.

\section{Appendix A. Second Order Sliding Mode Power Control}

As indicated in Section 3, a SOSM controller is used in this paper, although First Order Sliding Mode (FOSM) controllers could also be considered. In particular, SOSM algorithms have been widely accepted to control power system, due to their robust features while providing chattering amelioration [26]. It is not the objective of this paper to describe the controller design in depth, but an outline is provided in this Appendix.

A well-known dynamical averaged model of the power module based on boost converter with second-order low-pass filter (see Figure 10) can be described by:

$$
\dot{x}=f(x)+h(x) u \quad \text { with } \quad x=\left[\begin{array}{lll}
i_{s} & v & i_{c}
\end{array}\right]^{\prime}
$$

where

$$
f(x)=\left[\begin{array}{c}
-\frac{1}{L_{f}} v-\frac{R_{s} i_{s}}{L_{f}}+\frac{V_{o c}}{L_{f}} \\
\frac{1}{C_{f}} i_{s}-\frac{1}{C_{f}} i_{c} \\
\frac{1}{L_{b}} v-\frac{R_{b}}{L_{b}} i_{c}
\end{array}\right], \quad h(x)=\left[\begin{array}{c}
0 \\
0 \\
-\frac{V_{d c}}{L_{b}}
\end{array}\right] .
$$

The power module controller is designed to regulate the delivered electrical power $P_{f}$ to the DC bus.

To developed the SM controller, firstly, the control objective must be defined through the sliding variable $\sigma$ as:

$$
\sigma(x)=v i_{c}-P_{\text {fref }} .
$$

Then, the SOSM control objective will be accomplished when system trajectories reach and remain on the second-order sliding surface $\sigma=\dot{\sigma}=0$.

Please note that from (A3), the sliding variable $\sigma$ has relative degree one with respect to the duty cycle $u$. Then, a Super-Twisting Algorithm (STA) is employed, because of its direct application to relative degree one systems.

The SOSM-STA controller structure is defined as:

$$
\begin{aligned}
u & =-\alpha \cdot|\sigma(x)|^{1 / 2} \cdot \operatorname{sign}(\sigma(x))+\omega \\
\dot{\omega} & =-\beta \cdot \operatorname{sign}(\sigma(x))
\end{aligned}
$$

where $\alpha$ and $\beta$ are the STA gains. They are computed from

$$
\ddot{\sigma}(x, u)=\varphi(x, u)+\gamma(x) \cdot u
$$

by bounding functions $\varphi(x, u)$ and $\gamma(x)$ (see [26,27] for more details). Then, once the SOSM controlled system reaches $\sigma=0$, the resulting ZD will be the one described in Section 2.1.2.

\section{References}

1. Dragičević, T.; Lu, X.; Vasquez, J.C.; Guerrero, J.M. DC Microgrids-Part II: A Review of Power Architectures, Applications, and Standardization Issues. IEEE Trans. Power Electron. 2016, 31, 3528-3549, doi:10.1109/TPEL.2015.2464277. [CrossRef] 
2. Waqar, A.; Shahbaz Tanveer, M.; Ahmad, J.; Aamir, M.; Yaqoob, M.; Anwar, F. Multi-Objective Analysis of a CHP Plant Integrated Microgrid in Pakistan. Energies 2017, 10, 1625, doi:10.3390/en10101625. [CrossRef]

3. Gabriel Rullo, P.; Costa-Castelló, R.; Roda, V.; Feroldi, D. Energy Management Strategy for a Bioethanol Isolated Hybrid System: Simulations and Experiments. Energies 2018, 11, 1362, doi:10.3390/en11061362. [CrossRef]

4. Werth, A.; Kitamura, N.; Matsumoto, I.; Tanaka, K. Evaluation of centralized and distributed microgrid topologies and comparison to Open Energy Systems (OES). In Proceedings of the 2015 IEEE 15th International Conference on Environment and Electrical Engineering (EEEIC), Rome, Italy, 10-13 June 2015; pp. 492-497, doi:10.1109/EEEIC.2015.7165211. [CrossRef]

5. Li, S.; Yu, X.; Fridman, L.; Man, Z.; Wang, X. Advances in Variable Structure Systems and Sliding Mode Control-Theory and Applications; Springer: New York, NY, USA, 2018; Volume 115.

6. Fridman, L.; Barbot, J.P.; Plestan, F. Recent Trends in Sliding Mode Control; IET: London, UK, 2016.

7. Bandyopadhyay, B.; Sivaramakrishnan, J.; Spurgeon, S. Advances in Sliding Mode Control. Concept, Theory and Implementation; Springer: New York, NY, USA, 2013; Volume 440.

8. Fridman, L.; Moreno, J.; Iriarte, R. Sliding Modes after the first Decade of the 21st Century; Springer: New York, NY, USA, 2011.

9. Bartolini, G.; Fridman, L.; Pisano, A.; Usai, E. Modern Sliding Mode Control Theory. New Perspectives and Applications; Springer: New York, NY, USA, 2008; Volume 375.

10. Martínez-Treviño, B.; Jammes, R.; Aroudi, A.; Martinez-Salamero, L. Sliding-mode control of a boost converter supplying a constant power load. IFAC-PapersOnLine 2017, 50, 7807-7812, doi:10.1016/j.ifacol.2017.08.1055. [CrossRef]

11. Yasin, A.R.; Ashraf, M.; Bhatti, A.I. Fixed Frequency Sliding Mode Control of Power Converters for Improved Dynamic Response in DC Micro-Grids. Energies 2018, 11, 2799, doi:10.3390/en11102799. [CrossRef]

12. Ramos-Paja, C.A.; Bastidas-Rodríguez, J.D.; González, D.; Acevedo, S.; Peláez-Restrepo, J. Design and Control of a Buck-Boost Charger-Discharger for DC-Bus Regulation in Microgrids. Energies 2017, 10, 1847, doi:10.3390/en10111847. [CrossRef]

13. Serna-Garcés, S.I.; Gonzalez Montoya, D.; Ramos-Paja, C.A. Sliding-Mode Control of a Charger/Discharger DC/DC Converter for DC-Bus Regulation in Renewable Power Systems. Energies 2016, 9, 245, doi:10.3390/en9040245. [CrossRef]

14. Su, X.; Han, M.; Guerrero, J.M.; Sun, H. Microgrid Stability Controller Based on Adaptive Robust Total SMC. Energies 2015, 8, 1784-1801, doi:10.3390/en8031784. [CrossRef]

15. Singh, S.; Gautam, A.; Fulwani, D. Constant power loads and their effects in DC distributed power systems: A review. Renew. Sustain. Energy Rev. 2017, 72, 407-421, doi:10.1016/j.rser.2017.01.027. [CrossRef]

16. AL-Nussairi, M.K.; Bayindir, R.; Padmanaban, S.; Mihet-Popa, L.; Siano, P. Constant Power Loads (CPL) with Microgrids: Problem Definition, Stability Analysis and Compensation Techniques. Energies 2017, 10, 1656, doi:10.3390/en10101656. [CrossRef]

17. Huangfu, Y.; Pang, S.; Nahid-Mobarakeh, B.; Guo, L.; Rathore, A.K.; Gao, F. Stability Analysis and Active Stabilization of On-board DC Power Converter System with Input Filter. IEEE Trans. Ind. Electron. 2018, 65, 790-799, doi:10.1109/TIE.2017.2703663. [CrossRef]

18. Mishra, R.; Hussain, M.N.; Agarwal, V. A Sliding Mode Control based stabilization solution for multiple Constant Power Loads with identical input filters interfaced with the DC bus of a 'More Electric' Aircraft. In Proceedings of the 2016 IEEE International Conference on Power Electronics, Drives and Energy Systems (PEDES), Trivandrum, India, 14-17 December 2016; pp. 1-6, doi:10.1109/PEDES.2016.7914300. [CrossRef]

19. Li, A.; Zhang, D. Necessary and sufficient stability criterion and new forbidden region for load impedance specification. Chin. J. Electron. 2014, 23, 628-634,

20. Wu, M.; Lu, D.D.C. Active stabilization methods of electric power systems with constant power loads: A review. J. Mod. Power Syst. Clean Energy 2014, 2, 233-243. doi:10.1007/s40565-014-0066-y. [CrossRef]

21. Riccobono, A.; Santi, E. Comprehensive Review of Stability Criteria for DC Power Distribution Systems. IEEE Trans. Ind. Appl. 2014, 50, 3525-3535, doi:10.1109/TIA.2014.2309800. [CrossRef]

22. Riccobono, A.; Santi, E. A novel Passivity-Based Stability Criterion (PBSC) for switching converter DC distribution systems. In Proceedings of the 2012 Twenty-Seventh Annual IEEE Applied Power Electronics Conference and Exposition (APEC), Orlando, FL, USA, 5-9 February 2012; pp. 2560-2567, doi:10.1109/APEC.2012.6166184. [CrossRef] 
23. Sanchez, S.; Molinas, M. Assessment of a stability analysis tool for constant power loads in DC-grids. In Proceedings of the 2012 15th International Power Electronics and Motion Control Conference (EPE/PEMC), Novi Sad, Serbia, 4-6 September 2012; pp. DS3b.2-1-DS3b.2-5, doi:10.1109/EPEPEMC.2012.6397317. [CrossRef]

24. Sanchez, S.; Ortega, R.; Griño, R.; Bergna, G.; Molinas, M. Conditions for Existence of Equilibria of Systems With Constant Power Loads. IEEE Trans. Circuits Syst. I Regul. Pap. 2014, 61, 2204-2211, doi:10.1109/TCSI.2013.2295953. [CrossRef]

25. Miyagi, H.; Munda, J.L.; Miyagi, N. Study on Lyapunov Functions for Lienard-type Nonlinear Systems. IEEJ Trans. Electron. Inf. Syst. 2001, 121, 748-755.

26. Levant, A. Principles of 2-sliding mode design. Automatica 2007, 43, 576-586. [CrossRef]

27. Shtessel, Y.; Edwards, C.; Fridman, L.; Levant, A. Sliding Mode Control and Observation; Springer: New York, NY, USA, 2014.

(C) 2019 by the authors. Licensee MDPI, Basel, Switzerland. This article is an open access article distributed under the terms and conditions of the Creative Commons Attribution (CC BY) license (http:/ / creativecommons.org/licenses/by/4.0/). 